\title{
Limb Ischemic Preconditioning or Dezocine Pre-Treatment will Reduce Acute Gastric Mucosal Injury in Rats
}

\author{
Zhiming Zhang, Huidan Ou Yang, Wenge Yuan, Yuwen Wang and Wei Hong Dou* \\ Department of Anesthesiology and Operating-room, Chenzhou No.1 peoples' hospital, South China University, China
}

*Corresponding author: Weihong Dou, Department of Anesthesiology and Operating-room, Chenzhou No.1 peoples' hospital,

South China University, Chenzhou City, Hunan Province, 423000, China

\section{ARTICLE INFO}

Received: 蔧 January 04, 2021

Published: 蔧 March 01, 2021

Citation: Zhiming Zhang, Huidan OuYang, Wenge Yuan, Yuwen Wang, WeiHong Dou. Limb Ischemic Preconditioning or Dezocine Pre-Treatment will Reduce Acute Gastric Mucosal Injury in Rats. Biomed J Sci \& Tech Res 34(2)-2021. BJSTR. MS.ID.005529.

Keywords: Remote Ischemic Precondition; Dezocine Pre-Treatment; Acute Stress; Gastric Mucosal Injury

Abbreviations: SD: Sprague Dawley; GI: Gastric Injury; LIPc: Limb Ischemic Preconditioning; SOD: Superoxide Dismutase; MPO: Myeloperoxidase; MOR: Mu Opioid Receptor

\section{ABSTRACT}

Objective: The proposal of the studying was to investigate the protective effect of Limb ischemic precondition or Dezocine pre-treatment in acute stress-related gastric mucosal injury.

Methods: Forty-five Sprague Dawley (SD) male rats at 2-3 month were randomly divided into three groups: controlled group (Group $C, n=15$ ), Ischemic precondition group (Group T, $\mathrm{n}=15$ ): rats in this group received 5 cycles of ischemic precondition of 5-5 min in their left legs; Dezocine pre-treatment group (Group D, $n=15$ ): Rats in this group received Dezocine subcutaneous injection at a dosage of $10 \mu \mathrm{g} / \mathrm{g}$. Rats were beheaded after $6 \mathrm{~h}$ of cold water immerse, and stomach was taken out for Gastric injury index (GI), HE stains, and measurement of MDA, SOD, MPA.

\section{Result:}

(1) There were significant lower GI scores and less Inflammatory response in gastric (fewer Neutrophils) in Group T and Group D when compared to Group C, $(\mathrm{p}<0.05$, $\mathrm{CI}=95 \%)$;

(2) There levers of MDA and MPA were significantly lower in Group T and Group D when compared to That of Group C, $(\mathrm{p}<0.05, \mathrm{CI}=95 \%)$ and levers of SOD in Group T and Group D were significantly higher than that of Group C, $(\mathrm{p}<0.05, \mathrm{CI}=95 \%)$;

(3) Lever of SOD in Group T was higher than that in Group D, (P=0.048, CI=95\%).

Conclusion: Both Limb ischemic preconditioning and Dezocine pre-treatment will reduce acute gastric mucosal injury by inhibiting the inflammatory response and cleaning oxygen radical.

\section{Introduction}

Acute gastric mucosal lesions refer to acute inflammation of the gastric mucosa [1,2], Symptoms including gastric mucosal congestion, edema, and erosion [3-5]. Its main feature is the superficial injury of the gastric mucosa [1]. There are many reasons for gastric mucosal lesions. The main cause is considered as: Under the circumstance of stress [1,6], the inner body of animals or humans will redistribute blood flow, resulting in a decreased gastric mucosal blood perfusion, leading to ischemic necrosis [7]. If reperfusion occurs, the ischemia-reperfusion injury will occur $[6,8]$. Under severe stress, such as severe trauma, burns, and severe cranial trauma, gastric mucosal ischemia can be very severe and even massive bleeding, leading to poor treatment and delaying recovery of patients $[1,8]$. Opioids are frequently used for clinical analgesic treatment and intraoperative stress regulation. Studies have shown that the prophylactic use of opioids can reduce the stress state of patients in surgery, attenuating ischemiareperfusion in heart $[9,10]$, kidney [11-13], liver [14] and gastric mucous membrane, leading to a reduction in stress-related injuries, including gastric mucosal damage. Studies have reported that pretreatment with fentanyl or sufentanil before stress attenuates ischemia-reperfusion damage $[9,10,13,14]$. 
Among them, K opioid receptors may have an important role. Dezocine, a weak agonist of $\mathrm{u}$ opioid receptors and a strong agonist of $\mathrm{K}$ opioid receptors, is used prophylactically before stress or has some effect in preventing cerebral ischemia-reperfusion [15]. Studies also have found that by short-term ischemia-reperfusion treatment of organs or tissues far away from the target organ $[16,17]$, the same protective effect as the direct pre-treatment of the target organ can be achieved. A series of studies have shown that the ischemic preconditioning of the distal limbs has a certain protective effect on the distal organs, such as heart [18], lung, liver, kidney [19], and brain [18,20,21]. The main mechanism of the protective effect of Limb ischemic preconditioning (LIPc) may include improving liver energy metabolism and microcirculation, reducing free radical production, inhibiting the activation of inflammatory cells and apoptosis, and inducing the release of endogenous protective substances [8,12,14,22,23]. We speculate that LIPc can also have some protective effects on acute gastric mucosal lesions. In this article, We designed a comparison between LIPc and Dezocine Pre-treatment in rats to observe their protective effects on gastric mucosa under acute stress.

\section{Materials and Methods}

\section{Grouping}

After the approval of the Animal Ethics Committee in our hospital. 45 clean Sprague Dawley (SD) male rats at age 2-3 month were randomly assigned to the control group (group $\mathrm{C}, \mathrm{n}=15$ ), the ischemia-reperfusion group (group $\mathrm{T}, \mathrm{n}=15$ ) and the Dezocine pretreatment group (Group D, n=15).

\section{Limb Ischemic Preconditioning}

Rats are induced with isoflurane inhalation and continue to inhale $2 \%$ to maintain anesthesia. The rat was fixed to a self-made rat holder with four limbs fully stretched the root of the right thigh was tied with a bandage until the femoral artery pulsation disappeared (blood flow was blocked) and the sole of the foot showed ischemic signs. After blood flow was blocked for $5 \mathrm{~min}$, a bandage was release for $5 \mathrm{~min}$, one block time and one release time was considered as one cycle of LIPc. LIPc was performed when the rat was anaesthetized.

\section{Dezocine Pre-Treatment}

After rat shoulder was picked up by index finger and thumb grasping and alcohol disinfected the rat shoulder skin three times, $10 \mu \mathrm{g} / \mathrm{g}$ of dezocine was injected into the skin base using a $25 \mathrm{~g}$ injection needle. After the injection was finished, the kneading was lightly pressed to make the group mark and placed inside a ventilated cage. And they were Waiting for the next operation.

\section{Acute Stress Model}

All the pre-treated rat as inducing with isoflurane in isoflurane inhale jam. After induced, the rat was fixed to a self-made moveable rats' holder with four limbs stretched. After the rat was fully recovered from Isoflurane, it was immersed into a 4 degrees Celsius water tank with the water level to the rat neck for $6 \mathrm{~h}$ and then taken out.

\section{Samples Collection}

The rats were killed by decapitation, the abdominal cavity was opened, and the stomach was taken out. The stomach was cut along the greater curvature of the stomach and then laid flat, washed with cold saline, and the gastric mucosa was observed under a $10 \mathrm{x}$ microscope. Assessing gastric mucosal injury index (GI) according to Guth standard [24]: spotting bleeding point 1 point, erosion $1 \mathrm{~cm}$ 2 points, erosion $1 \sim 2 \mathrm{~cm} 3$ points, erosion $2 \sim 3 \mathrm{~cm} 4$ points, erosion $3 \mathrm{~cm} 5$ points, $2 \mathrm{~mm}$ width Score 2 , all scores are added up as GI score. After GI scored, a soy-sized stomach tissue was fixed in $4 \%$ neutral formaldehyde, embedded in paraffin, and stained with (Hematoxylin and Eosin) HE, and then histological examination was performed. Malondialdehyde (MDA), Superoxide Dismutase (SOD) and Myeloperoxidase (MPO) were measured by the colorimetric method with analysis kits (Jiancheng, Nanjing, China).

\section{Statistics Analysis}

GraphPad Prism 8(San Diego, CA, USA) was selected as statistical analysis software. GI scores were presented as Median (range), MDA, SOD and MPO were presented as Mean \pm SD. Oneway ANOVA test was used for data conforming to the normal distribution, while the Nonparametric (Mann-Whitney) test is used for non-normally distributed data. $\mathrm{P}<0.05, \mathrm{CI}=95 \%$ was considered statistically significant.

\section{Result}

All the animals were beheaded with a sharp knife after isoflurane inhaled, all data was collected follow the plan following the research flow chart, see Figure 1. There was no significant difference between 3 groups at Ages and Weights.

\section{Histological Examination}

More bleeding points can be noticed with bare eyes in Group C when compared to that in Group T and Group D, showing in Figures 2A-2D. GI scores under 100X microscope in group C is (10.68 \pm 4.87), which is significantly higher than that in Group T (5.8 \pm 2.21$)$, and Group D $(5.6 \pm 2.19), \mathrm{P}<0.05, \mathrm{CI}=95 \%$, showing in Figure $2 \mathrm{E}$. More Neutrophils can be sighed in HE stained slice, showing in Figures 2D-2J. 


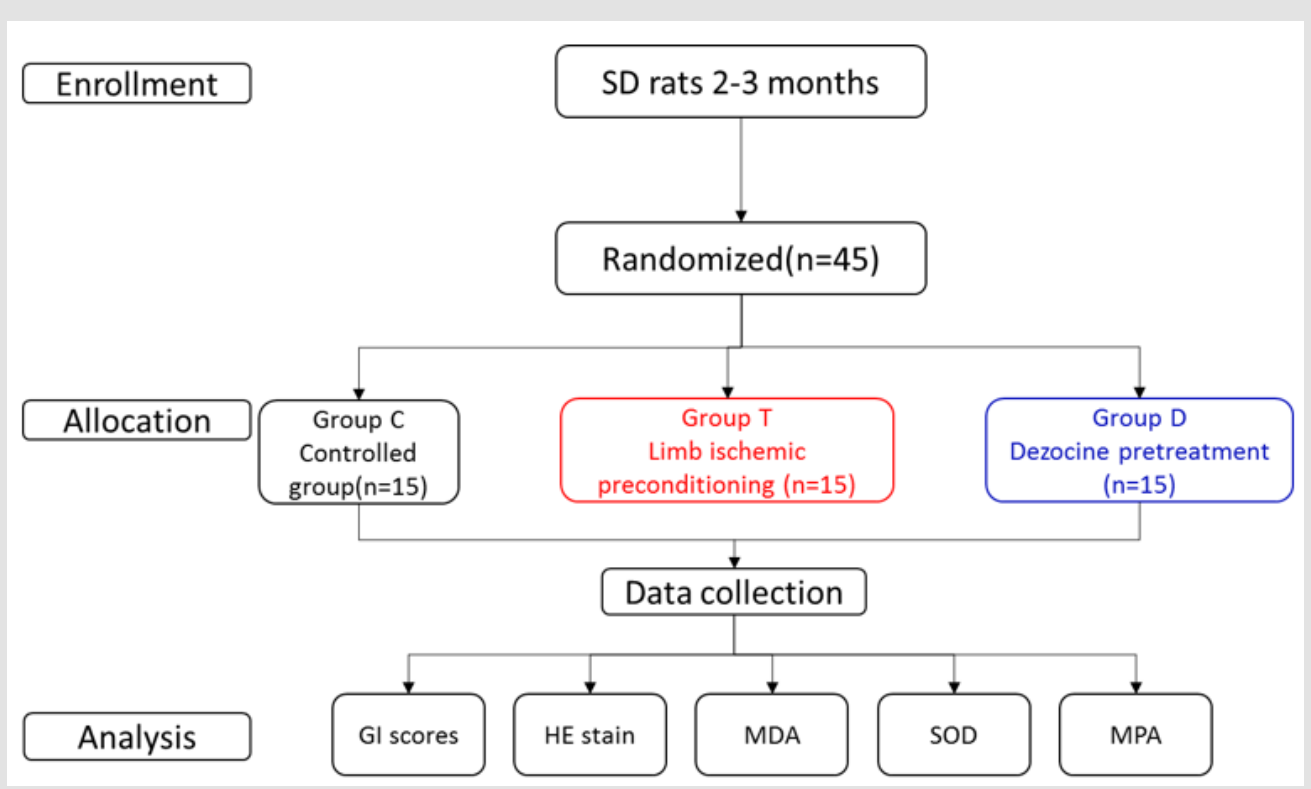

Figure 1: Research flow chart.

GI; Mucosal Injury Index; MDA: Malondialdehyd; SOD: Superoxide Dismutase; MPA: Myeloperoxidase
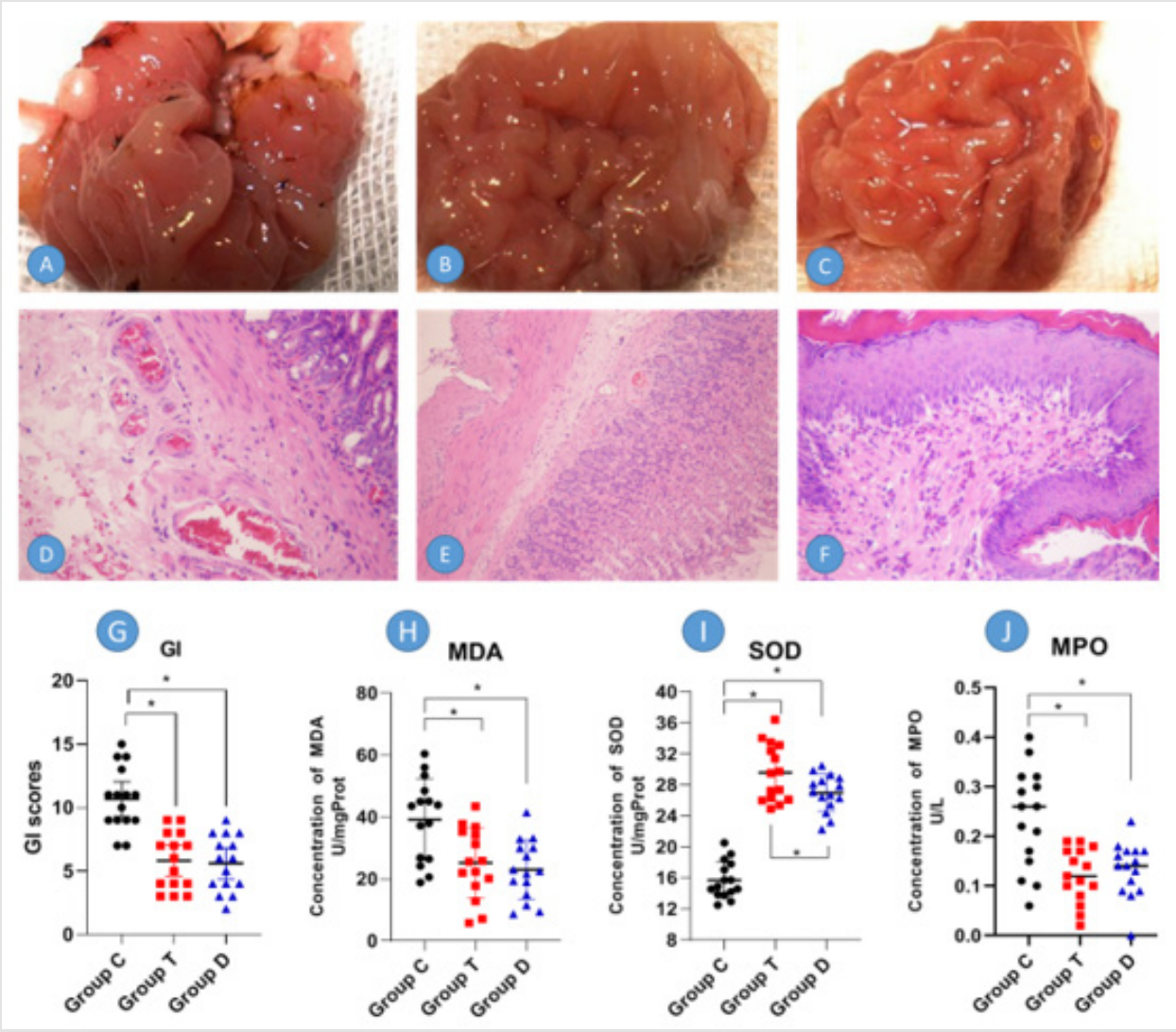

Figure 2: Histological examination of gastric tissue and laboratory test results.

A, B, C showing the gastric mucosal pathological changes under bare eyes, A Group C, B Group T, C group D, respectively. E, F, G showing the pathological difference under 100x microscope with HE stain in Group C, Group T and Group D, respectively. Compared with $\mathrm{D}$, gastric mucosal morphology was more intact with fewer neutrophils in $\mathrm{E}, \mathrm{F}$.

GI; Mucosal Injury Index; MDA: Malondialdehyd; SOD: Superoxide Dismutase; MPA: Myeloperoxidase; *P<0.05 


\section{Laboratory Test Results}

With the precondition or pre-treatment of limb ischemic and Dezocine, there was a significantly lower lever of MDA and MPA in Group T and Group D, (P<0.05 CI=95\%), respectively. At the same time, compared to Group D, but no significant difference can be found between Group T and Group D. On the other hand, SOD in Group T and Group D was significantly higher than that in Group C, $(\mathrm{P}<0.05 \mathrm{CI}=95 \%)$, However, Group T showed a higher level of SOD when compare to Group D, (P=0.048, CI=95\%).

\section{Discussion}

The results of our research showed that limb ischemic precondition and dezocine pre-treatment can all had the potential of gastric mucosal lesions under acute stress, evidence including less bleeding point, Lower GI scores and less the inflammatory response around gastric mucosal. Research showed that remote ischemic precondition was a promising method for protecting organ Ischemia-reperfusion of many surgery, including cardiac surgery, liver surgery, esophagectomy, kidney transplanting, etc. [16-21,25]. In cardiac surgery and advocating its practicing in regular clinical activity [26]. Gastric mucosal was vulnerable to acute stress, such as cold water immersed for a long time( $>2 \mathrm{~h})$. research also show that remote precondition can have some effect on Gastric Mucosal. In this study, the low limb was selected as the remote ischemic precondition site because of its maneuverability in rats. In this study, after 5 cycle of LIPc, it shows promising protection of gastric mucosal.

Opioids were reported of having gastric mucosal in acute stress by several pieces of research [9,11-13]. The drugs including morphine, fentanyl and sufentanil. Mu opioid receptor (MOR) playing some roles in the protective effect [9-11,13], while k opioids receptor could play a more important role in the gastrointestinal system [27]. Dezocine was reported as weak MOR agonists and a potent KOR agonists $[15,28,29]$, which could be a better choice after gastrointestinal surgery in postoperative analgesia [30]. Results in this study showed that: Dezocine could also have an effective gastric mucosal protection under acute stress, similar to LIPc in GI, MDA and MPA, but lower SOD lever ( $\mathrm{P}=0.048, \mathrm{CI}=95 \%)$. This may relate to the pathway of these two methods: LIPc was evoking the potential anti-ischemia-reperfusion effects by increasing protective substance release by local tissues [26]. In comparison, opioids were protecting gastric mucosal of stress by Inhibiting of sympathetic nervous system [30].

The study's limitation includes no dose-effect relationship of dezocine and not the cycle-effect relationship of LIPc in gastric mucosal protection. More evidence could be introduced in further research could explain the mechanism of these two methods in gastric mucosal protection.

\section{Conclusion}

Both Lamp ischemic precondition in 5-5 min 5 cycles and dezocine pre-treatment can reduce acute gastric mucosal injury in rats.

\section{Funding and Acknowledgement}

This studying was funding by the following grant: Hunan Science and Technology Plan Project: 2019JJ40010, Chenzhou Science and Technology Plan Project:CZ2014018 and Chenzhou No.1 People's Hospital Scientific research project: N2014-012.

\section{Conflicts of Interest}

No conflicts of interest need to be declared.

\section{References}

1. Yoo JH, Park EJ, Kim SH, Lee HJ (2020) Gastroprotective Effects of Fermented Lotus Root against Ethanol/HCl-Induced Gastric Mucosal Acute Toxicity in Rats. Nutrients 12(3).

2. Yeo D, Hwang SJ, Kim WJ, Youn HJ, Lee HJ (2018) The aqueous extract from Artemisia capillaris inhibits acute gastric mucosal injury by inhibition of ROS and NF-kB. Biomed Pharmacother 99: 681-687.

3. Peña Mercado E, Garcia Lorenzana M, Beltran NE (2018) Histomorphometric analysis with a proposed tissue lesion index in ischemia-reperfusion induced gastric mucosa damage. Histol Histopathol 33(10): 1047-1058.

4. Peña Mercado E, Garcia Lorenzana M, Arechaga Ocampo E, González De la Rosa CH, Beltran NE (2018) Evaluation of HIF-1 $\alpha$ and iNOS in ischemia/reperfusion gastric model: bioimpedance, histological and immunohistochemical analyses. Histol Histopathol 33(8): 815-823.

5. Perveen R, Azmi MA, Zaidi IH, Syed Naeemul Hassan Naqvi, Syed Mohammad Mahmood, et al. (2013) Assessment of Cedrus deodara root oil on the histopathological changes in the gastrointestinal tissues in rats. Pak J Pharm Sci 26(3): 571-576.

6. Omayone TP, Salami AT, Olopade JO, Olaleye SB (2020) Attenuation of ischemia-reperfusion-induced gastric ulcer by low-dose vanadium in male Wistar rats. Life Sci 259: 118272.

7. Murakami S, Takayama F, Egashira T, Imao M, Mori A (2012) Protective Effect of Fermented Papaya Preparation on Stress-Induced Acute Gastric Mucosal Lesion. Journal of Biophysical Chemistry 3(4): 311-316.

8. Odukanmi OA, Salami AT, Ashaolu OP, Adegoke AG, Olaleye SB (2018) Kolaviron attenuates ischemia/reperfusion injury in the stomach of rats. Appl Physiol Nutr Metab 43(1): 30-37.

9. Tire Y, Sarkilar G, Esen H, Onoglu R, Uzun ST (2018) The effect of intrathecal sufentanil preconditioning against myocardial ischemiareperfusion injury. Bratisl Lek Listy 119(4): 240-244.

10.Xu Q Li QG, Fan GR, Liu QH, Mi FL, et al. (2017) Protective effects of fentanyl preconditioning on cardiomyocyte apoptosis induced by ischemia-reperfusion in rats. Braz J Med Biol Res 50(2): e5286.

11. Franco Acevedo A, Echavarria R, Moreno Carranza B, David Garcia, Ricardo Gonzalez, et al. (2020) Opioid Preconditioning Modulates Repair Responses to Prevent Renal Ischemia-Reperfusion Injury. Pharmaceuticals (Basel) 13(11).

12. Echavarría R, Garcia D, Figueroa F, Adriana Franco Acevedo, Julio Palomino, et al. (2020) Anesthetic preconditioning increases sirtuin 2 gene expression in a renal ischemia reperfusion injury model. Minerva Urol Nefrol 72(2): 243-249. 
13. Palomino J, Echavarria R, Franco Acevedo A, Moreno Carranza B, Melo Z (2019) Opioids Preconditioning Upon Renal Function and IschemiaReperfusion Injury: A Narrative Review. Medicina (Kaunas) 55(9).

14. Lian YH, Fang J, Zhou HD, Jiang HF, Xie KJ (2019) Sufentanil Preconditioning Protects Against Hepatic Ischemia-Reperfusion Injury by Suppressing Inflammation. Med Sci Monit 25: 2265-2273.

15. Gao C, Gao S, Zhao X, Fei WANG, Huixia WANG, et al. (2020) Effects of Dezocine on neuronal apoptosis induced by cerebral ischemia/ reperfusion. Minerva Med.

16. Liu ZB, Yang WX, Fu XH, Zhao LF, Gao JL (2015) Remote ischemic precondition prevents radial artery endothelial dysfunction induced by ischemia and reperfusion based on a cyclooxygenase-2-dependent mechanism. Int J Clin Exp Med 8(11): 20946-52.

17. Ulus AT, Yavas S, Sapmaz A, Ziysan Sakaoğullari, Erdal Simsek, et al. (2014) Effect of conditioning on visceral organs during indirect ischemia/reperfusion injury. Ann Vasc Surg 28(2): 437-444.

18. Kepler T, Kuusik K, Lepner U, Joel Starkopf, Mihkel Zilmer, et al. (2020) Remote Ischaemic Preconditioning Attenuates Cardiac Biomarkers During Vascular Surgery: A Randomised Clinical Trial. Eur J Vasc Endovasc Surg 59(2): 301-308.

19. Kasepalu T, Kuusik K, Lepner U, Joel Starkopf, Mihkel Zilmer, et al. (2020) Remote Ischaemic Preconditioning Reduces Kidney Injury Biomarkers in Patients Undergoing Open Surgical Lower Limb Revascularisation: A Randomised Trial. Oxid Med Cell Longev : 7098505.

20. Yang G, Yang Y, Li Y, Hu Z (2020) Remote liver ischaemic preconditioning protects rat brain against cerebral ischaemia-reperfusion injury by activation of an AKT-dependent pathway. Exp Physiol 105(5): 852-863.

21. Mustonen C, Honkanen HP, Lehtonen S, Hannu Tuominen, Tuomas Mäkelä, et al. (2020) Moderate hypothermia with remote ischaemic preconditioning improves cerebral protection compared to deep hypothermia: a study using a surviving porcine model. Eur J Cardiothorac Surg 58(2): 269-276.

\section{ISSN: 2574-1241}

DOI: 10.26717/BJSTR.2021.34.005529

Weihong Dou. Biomed J Sci \& Tech Res

(C) This work is licensed under Creative Commons Attribution 4.0 License

Submission Link: https://biomedres.us/submit-manuscript.php
22. Zhao ZH, Hao W, Meng QT, Du XB, Lei SQ, et al. (2017) Long non-coding RNA MALAT1 functions as a mediator in cardioprotective effects of fentanyl in myocardial ischemia-reperfusion injury. Cell Biol Int 41(1): 62-70.

23. Ohara R, Périco LL, Rodrigues VP, Gabriela Bueno, Ana Caroline Zanatta, et al. (2020) Terminalia catappa L. infusion accelerates the healing process of gastric ischemia-reperfusion injury in rats. J Ethnopharmacol 256: 112793

24. Varga J, Stasko P, Tóth S, Zuzana Jonecová, Jarmila Veselá, et al. (2010) Morphological and apoptotic changes in the intestinal mucosa and lung parenchyma after ischaemic/reperfusion injury of the jejunum. Acta Vet Hung 58(2): 243-256.

25. Pan J, Li X, Peng Y (2016) Remote ischemic conditioning for acute ischemic stroke: dawn in the darkness. Rev Neurosci 27(5): 501-510.

26. Hausenloy DJ, Yellon DM (2008) Remote ischaemic preconditioning: underlying mechanisms and clinical application. Cardiovasc Res 79(3): 377-386.

27. Wang YH, Chai JR, Xu XJ, Ru Feng Ye, Gui Ying Zan, et al. (2018) Pharmacological Characterization of Dezocine, a Potent Analgesic Acting as a $\kappa$ Partial Agonist and $\mu$ Partial Agonist. Sci Rep 8(1): 14087

28. Ma J, Zhang P, Zhang Y, Zong Chen, Wenqi Xin, et al. (2016) Effect of dezocine combined with propofol on painless gastroscopy in patients with suspect gastric carcinoma. J Cancer Res Ther 12(Supplement): C271-C273.

29. Wu FX, Babazada H, Gao H, Xi Ping Huang, Chun-Hua Xi, et al. (2019) Dezocine Alleviates Morphine-Induced Dependence in Rats. Anesth Analg 128(6): 1328-1335.

30. Liu R, Huang XP, Yeliseev A, Xi J, Roth BL (2014) Novel molecular targets of dezocine and their clinical implications. Anesthesiology 120(3): 714723.

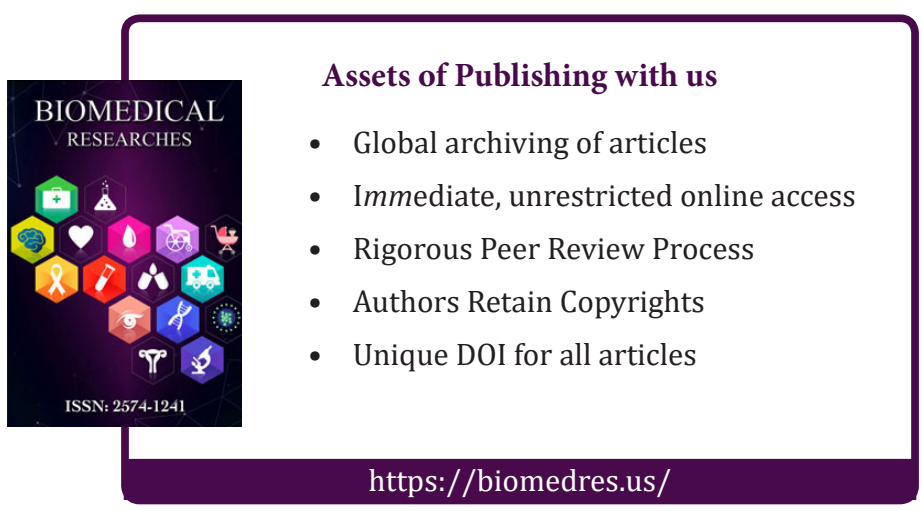

